VOL. 17 (1977), 125-134.

\title{
On families of finite sets no two of which intersect in a singleton
}

\section{Peter Frankl}

Let $X$ be a finite set of cardinality $n$, and let $F$ be a family of $k$-subsets of $X$. In this paper we prove the following conjecture of P. Erdös and V.T. Sós.

If $n>n_{0}(k), k \geq 4,|F|>\left(\begin{array}{l}n-2 \\ k-2\end{array}\right)$ then we can find two members $F$ and $G$ in $F$ such that $|F \cap G|=1$.

\section{Introduction and some lemmas}

Let $X$ be a finite set of cardinality $n$ and let $F$ be a family of $k$-subsets of $X$. Let us define

$$
F_{x}=\{F-x \mid x \in F \in F\} .
$$

We say that a family of sets is intersecting if any two members of it have non-empty intersection. Let $L$ be a set of non-negative integers. We say that $F$ is an $(n, L, k)$-system if, for any two different members $F, G$ of $F,|G \cap F| \in L$.

The Erdös-Ko-Rado Theorem (Erdös, Ko, and Rado [4]) states that if $F$ is an $(n,\{t, t+1, \ldots, k-1\}, k)$-system and $n>n_{0}(k)$; then $|F| \leq\left\{\begin{array}{l}n-t \\ k-t\end{array}\right)$ with equality holding if and only if for some t-element subset $Y$ of $X$, $F=\{F \subset X|| F \mid=k, Y \subseteq F\}$, where $t$ is a positive integer.

Erdös and Sós made the following conjecture (see Erdös [2]):

If $F$ is an $(n,\{0,2,3, \ldots, k-1\}, k)$-system, $k \geq 4, n \geq n_{0}(k)$,

Received 21 February 1977. 
then $|F| \leq\left(\begin{array}{l}n-2 \\ k-2\end{array}\right)$.

The aim of this paper is to prove this conjecture. For the case $k=4$ it was proved by Katona [5].

Obviously the condition is equivalent to that for every $x \in X, F_{x}$ is an intersecting family.

If $G$ is an intersecting family of $(k-1)$-subsets of $X$ then let us define

$$
\begin{aligned}
& G^{*}=\left\{E \subset X\left|E \neq \varnothing, \exists G_{1}, G_{2}, \ldots, G_{k}\right| E \mid\right. \\
&\text { such that } \left.G_{i} \cap G_{j}=E, 1 \leq i<j \leq k|E|\right\},
\end{aligned}
$$

$B(G)=\left\{B \in G^{*} \mid \not E \in G^{*}\right.$ such that $\left.E \varsubsetneqq B\right\}$.

From the definition it is evident that $G \subseteq G^{*}$ and consequently for every $G \in G$ there exists $B \in B(G)$ such that $B \subseteq G$. Therefore we call $B$ the $\Delta$-base of $G$.

A family $c=\left\{C_{1}, \ldots, C_{s}\right\}$ is called a $\Delta$-system of cardinality $s$ if for some set $K \varsubsetneqq C_{1}$ we have $C_{i} \cap C_{j}=K$ for $1 \leq i<j \leq s . K$ is called the kernel of the $\Delta$-system. Erdös and Rado [3] proved that there exists a function $f(k, s)$ such that any family consisting of $f(k, s)$ different $k$-sets contains a $\Delta$-system of cardinality $s$.

LEMMA 1. Let $F$ be an $(n,\{0,2,3, \ldots, k-1\}, k)$-system, $k \geq 4$, $x \in X, 1 \leq i \leq k-1$. Then we cannot find sets $B_{1}, \ldots, B_{k} \in B\left(F_{x}\right)$, forming a $\Delta$-system of cardinality $k^{i}$ and satisfying further $\left|B_{j}\right|=i+1$ for $1 \leq j \leq k^{i}$.

Proof. Let us suppose that for $B_{1}, \ldots, B_{k^{i}}$ the lemma fails; let $K$ be the kernel of the corresponding $\Delta$-system.

By the definition of the $\Delta$-base there exist sets $E_{j}^{r} \in F_{x}$ for $1 \leq r \leq k^{i}, 1 \leq j \leq k^{i+1}$, such that for $1 \leq j<j^{*} \leq k^{i+1}$, 
$E_{j}^{r} \cap E_{j^{*}}^{p}=B_{r}$. As the sets $E_{j}^{\perp}-B_{1}$ are pairwise disjoint and

$$
\left|\bigcup_{r=2}^{k^{i}} B_{r}\right|<k \cdot k^{i}=k^{i+1} \text {, }
$$

we can find a $j, 1 \leq j \leq k^{i+1}$ such that $E_{j}^{1}-B_{1}$ is disjoint to the union of the $B^{r^{\prime}} \cdot \mathbf{s}$. Let us define $D_{1}=E_{j}^{l}$. Let us suppose that $D_{r}$ is defined already for $r=1, \ldots, s-1$. Let us set

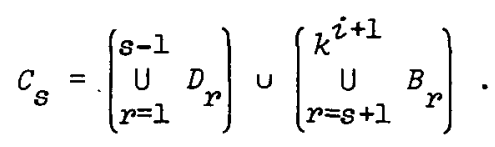

Then $\left|C_{s}\right|<k^{i+1}$.

Hence among the pairwise disjoint sets $E_{1}^{s}-B, \ldots, E_{k^{i+1}}^{s}{ }_{s}$ we can find one, say $E_{j}^{s}-B_{s}$, which is disjoint from $C_{s}$; let us define $D_{s}=E_{j}^{s}$. Let us continue this procedure until $s=k^{i}$. From the definition of the $D_{s}$ 's it follows that they belong to $F_{x}$, and that they form a $\Delta$-system of cardinality $k^{i} \geq k|K|$ with kernel $K$, yielding $K \in F_{x}^{*}$. But this is a contradiction as $K \varsubsetneqq B \in B\left(F_{x}\right)$. / /

In view of Lemma $1, B\left(F_{x}\right)$ contains at most $f\left(i+1, k^{i}\right)(i+1)-$ element sets for $i=1,2, \ldots, k-1$. Now the next lemma is obvious.

LEMMA 2. Let $F$ be an $(n,\{0,2,3, \ldots, k-1\}, k)$-system consisting of subsets of $X, x \in X, k \geq 4$. Suppose that $F_{x}^{*}$ does not contain any 1-element set and let $B_{1}, \ldots, B_{v}$ be the 2-element sets in it. Then

$$
\left|F_{x^{-}}\left\{E \in F_{x} \mid \exists j, 1 \leq j \leq v, B_{j} \subset E\right\}\right|<k \cdot f\left(k-1, k^{k-2}\right) \cdot\left(\begin{array}{l}
n-4 \\
k-4
\end{array}\right) .
$$

We need one more lemma.

LEMMA 3. Let $F$ be an $(n,\{0,2,3, \ldots, k-1\}, k)$-system 
consisting of subsets of $X$. Let $y, x$ be two not necessarily different elements of $X$. If $B \in B\left(F_{x}\right), C \in B\left(F_{y}\right)$, then $|(B \cup x) \cap(C \cup y)| \neq 1$.

Proof. If $B \in F_{x}, C \in F_{y}$, then the statement follows from the definition of $(n,\{0,2,3, \ldots, k-1\}, k)$-systems. So we may assume that, for example, $B \notin F_{x}$. By the definition of $B\left(F_{x}\right)$ there exist $F_{1}, \ldots, F_{k} \in F$ forming a $\Delta$-system with kernel $B \cup x$. As the sets $F_{i}-(B \cup x)$ are pairwise disjoint and in the case $(B \cup x) \cap(C \cup y)=1$, $|(C \cup y)-(B \cup x)| \leq k-1$, we can find an index $j, 1 \leq j \leq k$, such that $\left|F_{j} \cap(C \cup y)\right|=1$. If $(C \cup y) \in F$ then this is a contradiction to the definition of $(n,\{0,2,3, \ldots, k-1\}, k)$-systems. If $c \notin F$ then by the definition of $B\left(F_{y}\right)$ there exist $G_{1}, G_{2}, \ldots, G_{k} \in F$ which form a $\Delta$-system with kernel $C \cup y$. As the sets $G_{i}-(C \cup y)$ are pairwise disjoint and $\left|F_{j}-(C \cup y)\right|=k-1$, we can find an index $i$, $1 \leq i \leq k$, such that $\left(F_{j}-(C \cup y)\right) \cap G_{i}=\emptyset$; that is, $\left|F_{j} \cap G_{i}\right|=1$, a contradiction which proves the lemma.

\section{The proof of the result}

Let us first prove a slightly weaker result which, however, implies the conjecture of Erdös-Sós.

THEOREM 1. Let $F$ be an $(n,\{0,2,3, \ldots, k-1\}, k)$-system consisting of subsets of $X, n>n_{0}(k)$. Then one of the following cases occurs:

(i) $|F|<\left(\begin{array}{l}n-2 \\ k-2\end{array}\right)$;

(ii) there exist $x \neq y \in X$ such that

$$
F=\{F \subset X|| F \mid=k, x \in F, y \in F\} ;
$$

(iii) there exists $x \in X$ such that $\left|F_{x}\right|<\left(\begin{array}{l}n-3 \\ k-3\end{array}\right)$;

(iv) there exist $x \neq y \in X$ such that 


$$
|\{F \in F \mid\{x, y\} \cap F \neq \varnothing\}|<\left(\begin{array}{l}
n-3 \\
k-3
\end{array}\right)+\left(\begin{array}{l}
n-4 \\
k-3
\end{array}\right) .
$$

Proof. Let us argue indirectly. By Lemma 2 we may assume that for for every $x \in X, B\left(F_{x}\right)$ contains a set of cardinality at most 2 . If it contains a l-element set, say $\{y\}$, then the intersection property implies $B\left(F_{x}\right)=\{\{y\}\}$ and conversely $B\left(F_{y}\right)=\{\{x\}\}$. Indeed, if for some $F \in F,|F \cap\{x, y\}|=1$ holds then let us consider a $\Delta$-system of cardinality $k$ and with kernel $\{x, y\}$, consisting of members of $F$ such a system exists by the definition of $B\left(F_{x}\right)$. Now as $|F-\{x, y\}|=k-1$, there is a member of the $\Delta$-system, say $G$, which is disjoint from it; that is, $|F \cap G|=1$, a contradiction.

Now let us suppose that $B\left(F_{x}\right)$ consists of sets of cardinality at least 2 , and let $B_{1}, \ldots, B_{v}$ be the 2-element sets belonging to it. By Lemma 3 the $B_{i}$ 's form an intersecting family of 2-sets, and by Lemma 1 this family does not contain a $\Delta$-system of cardinality $k$. As $k \geq 4$, it follows $v<k$. Now Lemma 2 and $\left|F_{x}\right| \geq\left(\begin{array}{l}n-3 \\ k-3\end{array}\right)$ imply, for $n>n_{0}(k)$, that there exists an $i, 1 \leq i \leq v$, such that

$$
\left|\left\{G \in F_{x} \mid B_{i} \subset G\right\}\right|>1 / k\left(\begin{array}{l}
n-3 \\
k-3
\end{array}\right) \text {. }
$$

By symmetry reasons we may assume that (1) holds for $i=1$. Let us suppose first that for some $G \in F_{x}, G \nsupseteq B_{1}$ holds.

If $G \cap B_{1}=\emptyset$ then let us choose $k$ sets $G_{1}, \ldots, G_{k}$ belonging to $F_{x}$ and forming a $\Delta$-system with kernel $B_{1}$. Then $\left|G-B_{1}\right|=k-1$ implies that $G$ is disjoint from at least one of the $G_{i}$ 's, say from $G_{u}$. As $F_{x}$ is an intersecting family of sets, we see that $G \cap B_{1}=\emptyset$ is impossible. Hence if $G \not B_{1}$ then $\left|G \cap B_{1}\right|=1$. We prove now that this is impossible, too.

Let us define $H=G \cup B_{1} \cup \ldots \cup B_{v}$, and

$$
E_{1}=\left\{F-B_{1} \mid B_{1} \subseteq F \in F_{x},\left(F-B_{1}\right) \cap H=\emptyset\right\}
$$


From (1), and $n>n_{0}(k)$ it follows that, for example,

$$
\left|E_{1}\right|>1 / 2 k\left(\begin{array}{l}
n-3 \\
k-3
\end{array}\right)
$$

Hence there exists an element $z$ of $X-\left(B_{1} \cup x\right)$ which satisfies

$$
\left|\left\{E \in E_{1} \mid z \in E\right\}\right|>I / 2 k\left(\begin{array}{l}
n-4 \\
k-4
\end{array}\right) \text {. }
$$

In the case $k=4$ we just choose $z=E-\left(B_{1} \cup x\right)$ for some $E \in E_{1}$. By a result of Erdös [1] if a family 0 of s-subsets of $X$, $s \geq 1$, does not contain $f(s)$ pairwise disjoint members, then $|D|=o\left(\left(\begin{array}{c}n \\ s-1\end{array}\right)\right)$.

We apply this theorem for $D=\left\{E-z \mid E \in E_{1}, z \in E\right\}, s=k-4$, $f(s)=s+4$, to prove that there exist $k$ members of $E_{1}$, say $C_{1}, \cdots, C_{k}$, such that $C_{i} \cap C_{j}=\{z\}, I \leq i<j \leq k$. In the case $k=4$ we can choose $c_{1}=\ldots=c_{k}=\{z\}$.

Let $B$ be a member of $B\left(F_{z}\right)$ for which $\left|\left\{F \in F_{z} \mid B \subseteq F\right\}\right|$ is maximal. Then, as we proved it already for $x$, it follows that

$$
\left|\left\{F \in F_{z} \mid B \subset F\right\}\right|>1 / k\left(\begin{array}{l}
n-3 \\
k-3
\end{array}\right) \text {. }
$$

From Lemma 3 we know that $(B \cup z) \cap\left(B_{1} \cup x\right)=1$ is impossible. We prove now that these two sets cannot be disjoint either. Otherwise from a $\Delta$-system $F_{1}, \ldots, F_{k}$ consisting of members of $F$, and having kernel $B \cup z$, we could choose a set, say $F_{i}$, satisfying $F_{i} \cap\left(B_{1} \cup x\right)=\emptyset$. But then there is an index $j, 1 \leq j \leq k$, such that $C_{j} \cap F_{i}=\{z\}$. Now setting $G_{j}=\left(C_{j} \cup B_{1} \cup x \cup z\right) \in F,\left|G_{j} \cap F_{i}\right|=1$ is a contradiction, proving $(B \cup z) \cap\left(B_{1} \cup x\right) \neq \emptyset$.

$$
\begin{aligned}
& \text { As }|B| \leq 2 \text {, it follows now that }|B|=2 \text { and } B \subset\left(B_{1} \cup x\right) \text {. } \\
& \text { If } B=B_{1} \text { then from a } \Delta \text {-system } F_{1}^{\prime}, \ldots, F_{k} \text { consisting of members }
\end{aligned}
$$
of $F$ and having kernel $B \cup z$ we can choose a set, say $F_{i}$, which is 
disjoint from $G-B$. But then we have $\left|F_{i} \cap(G \cup x)\right|=1$, a contradiction.

Hence $x \in B$. This in turn implies $((B \cup z)-x) \in B\left(F_{x}\right)$, a contradiction since $z \notin\left(B_{1} \cup \ldots \cup B_{v}\right)$. This final contradiction proves that $G \nsupseteq B_{1}$ is impossible; that is, for every $G \in F_{x}, G \supseteq B_{1}$ holds, and in particular $v=1$. Hence $B\left(F_{x}\right)=\left\{B_{1}\right\}$.

Let $B_{1}=\left\{y_{1}, y_{2}\right\}$. We assert that $B\left(F_{y_{1}}\right)=\left\{\left\{y_{2}\right\}\right\}$.

Otherwise it follows from the definition of $B\left(F_{y_{1}}\right)$ that $\left\{x, y_{2}\right\}$ is a member of it. Then repeating the argument applied to $x$ for $y_{1}$ we obtain that $B\left(F_{y_{1}}\right)=\left\{\left\{x, y_{2}\right\}\right\}$; that is, every member of $F$ which contains $y_{1}$ contains $x$ and $y_{2}$ as well. Consequently we have

$$
\begin{aligned}
\left|\left\{F \in F \mid F \cap\left\{x, y_{1}\right\} \neq \emptyset\right\}\right| \leq \mid\{F \subset X|| F \mid=k, & \left.\left\{x, y_{1}, y_{2}\right\} \subset F\right\} \mid \\
& =\left(\begin{array}{l}
n-3 \\
k-3
\end{array}\right)<\left(\begin{array}{l}
n-3 \\
k-3
\end{array}\right)+\left(\begin{array}{l}
n-4 \\
k-3
\end{array}\right),
\end{aligned}
$$

contradicting the indirect assumptions.

So far we have proved that for every $x \in X$ either there exists a $y \in X$ such that $B\left(F_{x}\right)=\{\{y\}\}, B\left(F_{y}\right)=\{\{x\}\}$, or there exist $y, z \in X$ such that $B\left(F_{x}\right)=\{\{y, z\}\}, B\left(F_{y}\right)=\{\{z\}\}, B\left(F_{z}\right)=\{\{y\}\}$.

Now let $\left\{x_{1}, y_{1}\right\}, \ldots,\left\{x_{w}, y_{w}\right\}$ be the collection of all the different unordered pairs satisfying $x_{i}, y_{i} \in X, B\left(F_{y_{i}}\right)=\left\{\left\{x_{i}\right\}\right\}$, $B\left(F_{x_{i}}\right)=\left\{\left\{y_{i}\right\}\right\}, 1 \leq i \leq w$. By Lemma 3 all the elements $x_{i}, y_{i}$ are different; that is, they form $w$ pairwise disjoint 2-subsets of $X$. As we proved it is possible to divide the remaining elements of $X$ into $w$ classes $z_{1}, \ldots, z_{w}$ such that for $1 \leq i \leq w, z_{i} \in z_{i}$, we have $B\left(F_{z_{i}}\right)=\left\{\left\{x_{i}, y_{i}\right\}\right\}$. So we proved that $F$ is contained in the following family of subsets of $X$ : 
$F^{*}=\left\{F \subset X|| F \mid=k, F \cap\left(z_{i} \cup\left\{x_{i}, y_{i}\right\}\right) \neq \emptyset\right.$ implies

$$
\left.\left\{x_{i}, y_{i}\right\} \subset E, I \leq i \leq w\right\} \text {. }
$$

If $w=1$ then either (i) or (ii) holds. So we may assume $w \geq 2$. All we have to prove now is that in this case $|F *|<\left(\begin{array}{l}n-2 \\ k-2\end{array}\right)$.

We prove this by induction on $w$ and for every $n>k$.

By symmetry reasons we may assume that $\left|z_{1}\right| \leq\left|z_{2}\right|$. We count the number of members of $F^{*}$ according to the cardinality of their intersection with $z_{1} \cup\left\{x_{1}, y_{1}\right\}$. Let us define $n_{1}=\left|z_{1} \cup\left\{x_{1}, y_{1}\right\}\right|$. Then $\left|z_{1}\right| \leq\left|z_{2}\right|$ implies $n_{1} \leq n-n_{1}$. Using the induction hypothesis or the estimate for the case $w=1$, we obtain

$$
\left|F^{*}\right| \leq\left(\begin{array}{c}
n_{1}-2 \\
k-2
\end{array}\right)+\sum_{i=2}^{k-2}\left(\begin{array}{c}
n_{1}-2 \\
k-2-i
\end{array}\right)\left(\begin{array}{c}
n-n_{1}-2 \\
i-2
\end{array}\right)+\left(\begin{array}{c}
n-n_{1}-2 \\
i-2
\end{array}\right) \text {. }
$$

As

$$
\left(\begin{array}{c}
n_{I}-2 \\
k-2
\end{array}\right)=\left(\left(n_{I}-2\right) /(k-2)\right)\left(\begin{array}{c}
n_{I}-3 \\
k-3
\end{array}\right)<n_{I}\left(\begin{array}{c}
n-n_{1}-2 \\
k-3
\end{array}\right)
$$

and

$$
\left(\begin{array}{c}
n_{1}-2 \\
k-2-i
\end{array}\right) \leq\left(\begin{array}{c}
n_{1} \\
k-i
\end{array}\right), \quad i=2, \ldots, k-2,
$$

it follows from (4),

$$
\begin{aligned}
\left|F^{*}\right|<n_{1}\left(\begin{array}{c}
n-n_{1}-2 \\
k-3
\end{array}\right)+\sum_{i=2}^{k-2}\left(\begin{array}{c}
n_{1} \\
k-i
\end{array}\right)\left(\begin{array}{c}
n-n_{1}-2 \\
i-2
\end{array}\right) & +\left(\begin{array}{c}
n-n_{1}-2 \\
k-2
\end{array}\right) \\
& =\sum_{i=2}^{k}\left(\begin{array}{c}
n_{1} \\
k-i
\end{array}\right)\left(\begin{array}{c}
n-n_{1}-2 \\
i-2
\end{array}\right)=\left(\begin{array}{c}
n-2 \\
k-2
\end{array}\right) .
\end{aligned}
$$

THEOREM 2. Let $F$ be an $(n,\{0,2,3, \ldots, k-1\}, k)$-system, $k \geq 4$. Suppose that $n>n_{0}(k)+2\left(\begin{array}{c}n_{0}(k) \\ k\end{array}\right)$, where $n_{0}(k)$ is the bound from Theorem 1. Then either there exist two different elements $x, y$ such that $F=\{F \subset X|| F \mid=k,\{x, y\} \subset F\} \quad$ or $\quad|F|<\left(\begin{array}{l}n-2 \\ k-2\end{array}\right)$. 
Proof. Let us argue indirectiy and let $F$ be a counter-example. Let $|F|=\left(\begin{array}{l}n-2 \\ k-2\end{array}\right)+d$, where $d$ is a non-negative integer. We may apply

Theorem 1 to $F$. Hence either there exists $x \in X$ such that $\left|F_{x}\right|<\left(\begin{array}{l}n-3 \\ k-3\end{array}\right)$ or there exist two different elements $x, y$ in $X$ such that $|\{F \in F \mid F \cap\{x, y\} \neq \emptyset\}|<\left(\begin{array}{l}n-3 \\ k-3\end{array}\right)+\left(\begin{array}{l}n-4 \\ k-3\end{array}\right)$.

In the first case let us define $X_{1}=X-x$ and in the second $X_{1}=X-\{x, y\}$. In both cases we define $F_{1}=\left\{F \subset X_{1} \mid F \in F\right\}$. Then $F_{1}$ is an $\left(\left|x_{1}\right|,\{0,2,3, \ldots, k-1\}, k\right)$-system of cardinality at least

$$
\left(\begin{array}{c}
\left|x_{1}\right|-2 \\
k-2
\end{array}\right)+d+1
$$

Now we apply Theorem 1 to the family $F_{1}$, and we construct a set $X_{2}$ and a family of subsets, $F_{2}$, of $X_{2}$ such that $F_{2}$ is an $\left(\left|x_{2}\right|,\{0,2,3, \ldots, k-1\}, k\right)$-system of cardinality at least $\left(\begin{array}{c}\left|x_{2}\right|-2 \\ k-2\end{array}\right)+d+2$, and so on, and so on until we get a set $x_{p}$ and a family of $k$-subsets of $x_{r}, F_{r}$ such that $\left|x_{r}\right| \leq n_{0}(k)$.

Now the method of construction implies that

$$
\left|F_{p}\right| \geq\left(\begin{array}{c}
\left|x_{p}\right|-2 \\
k-2
\end{array}\right)+d+\left(\begin{array}{c}
n_{0}(k) \\
k
\end{array}\right)>\left(\begin{array}{c}
n_{0}(k) \\
k
\end{array}\right)
$$

a contradiction since the number of $k$-subsets of $X_{r}$ is $\left(\begin{array}{c}\left|x_{p}\right| \\ k\end{array}\right) \leq\left(\begin{array}{c}n_{0}(k) \\ k\end{array}\right)$

REMARK. One might conjecture that for an arbitrary integer $s$ and $k>k_{0}(s), n>n_{0}(k)$, any family of more than $\left(\begin{array}{l}n-s-1 \\ k-s-1\end{array}\right) \quad k$-subsets of an $n$-set contains two members intersecting in a set of cardinality $s$. The author can prove it only for $c_{k}\left(\begin{array}{l}n-s-1 \\ k-s-1\end{array}\right)$, where $c_{k}$ is a large constant 
depending only on $k$.

\section{References}

[1] P. Erdös, "A problem on independent r-tuples", Ann. Univ. Sci. Budapest. Eötvös Sect. Math. 8 (1965), 93-95.

[2] P. Erdös, "Problems and results in graph theory and combinatorial analysis", Proc. Fifth British Combinatomaz Conference, 1975, 169-172 (University of Aberdeen, Aberdeen, 1975. Congressus Numerantium, 15. Utilitas Mathematica, Winnipeg, 1976).

[3] P. Erdös and R. Rado, "Intersection theorems for systems of sets", J. London Math. Soc. 35 (1960), 85-90.

[4] P. Erdös, Chao Ko, and R. Rado, "Intersection theorems for systems of finite sets", Quart. J. Math. Oxford Ser. 12 (1961), 313-320.

[5] Gyula Katona (unpublished).

Magyar Tudományos Akadémia,

Matematikai Kutató Intézete,

Budapest,

Hungary. 